Short report

\title{
Transoral-transclival clipping of a midline vertebral artery aneurysm
}

\author{
EDWARD HITCHCOCK, RICHARD COWIE. \\ From the Department of Neurosurgery, University of Birmingham, and the Midland Centre for Neurosurgery \\ and Neurology, Smethwick, Warley, UK
}

SUMMARY The technical advantages and possible indications for a transoral-transclival approach for low midline posterior circulation aneurysms are discussed. A case report using such a route is described. The prognosis of untreated vertebro-basilar aneurysms is briefly discussed.

Vertebro-basilar aneurysms account for $5-10 \%$ of intracranial aneurysms. ${ }^{1-3}$ Studies of the natural history of patients treated without surgery indicate that there is a very high risk of recurrent haemorrhage in posterior fossa aneurysms. Hook et al ${ }^{4}$ followed up 16 unoperated cases of basilar aneurysm and of these 10 died of recurrent haemorrhage. Gillingham et $a l^{5}$ reported 11 deaths from haemorrhage in 16 patients treated without surgery. Twenty-five cases of basilar bifurcation aneurysms were reported by Troupp ${ }^{6}$ and of this total 13 died from re-bleeding up to 25 months from diagnosis.

The sub-temporal or Sylvian approaches are generally employed for aneurysms on the upper part of the basilar artery. ${ }^{7}$ Vertebro-basilar junction aneurysms however, are notoriously difficult of access. Drake $^{7}$ advises a posterior sub-occipital approach, even for aneurysms near the midline, combined with a transtentorial approach in certain cases. His description of the technique emphasises the difficulty of visualising the aneurysm and the need for considerable retraction of the medulla and vagal rootlets. For large or moderately-sized aneurysms with a laterally pointing fundus the approach is particularly difficult and dangerous, and in these circumstances the more direct transclival approach seems worthy of consideration. We report the successful obliteration of a vertebral artery aneurysm in a patient where the angiographic picture suggested

Address for reprint requests: Professor Edward Hitchcock, Midland Centre for Neurosurgery and Neurology, Holly Lane, Smethwick, Warley, West Midlands, B67 7JX, UK.

Received 17 September 1982. Accepted 13 November 1982 that a direct transclival approach would be necessary.

\section{Case report}

A 51-year-old right-handed woman suddenly collapsed with loss of consciousness whilst at a party. There were no preceding symptoms but on recovery she complained of severe, generalised headache, neck pain and photophobia and was admitted urgently to her local hospital with drowsiness, restlessness and vomiting. Bilateral fundal haemorrhages and papilloedema were noted without any other specific neurological deficit. Her father and two aunts had died from cerebral haemorrhage. A lumbar puncture was not performed and she was transferred to the Midland Centre for Neurosurgery and Neurology for further investigations the following day. The patient was drowsy, disorientated and very restless with slurred speech but no dysphasia. Apart from bilateral extensor plantar responses there were no other central nervous system abnormalities. She had considerable neck stiffness and a positive Kernig's sign, a pulse of $74 / \mathrm{min}$, blood pressure $150 / 95 \mathrm{~mm} \mathrm{Hg}$. A clinical diagnosis of subarachnoid haemorrhage was made confirmed by computed tomography which showed blood within the third and fourth ventricles and a superficial haematoma in the pontine and interpeduncular cisterns with acute hydrocephalus. Bilateral carotid and right vertebral angiograms on the day of admission revealed a large, apparently bi-lobed aneurysm at the origin of the right posterior-inferior cerebellar artery from the vertebral artery (fig $1 \mathrm{a}$ and b). Routine haematological and biochemical tests were normal and her clinical condition steadily improved over the following few days until, at the time of surgery, she was alert and orientated.

After careful consideration of the anatomical difficulties brought about by the midline position of the aneurysm due to the sweeping of the right vertebral artery to the left, bringing the fundus of the aneurysm immediately in front 

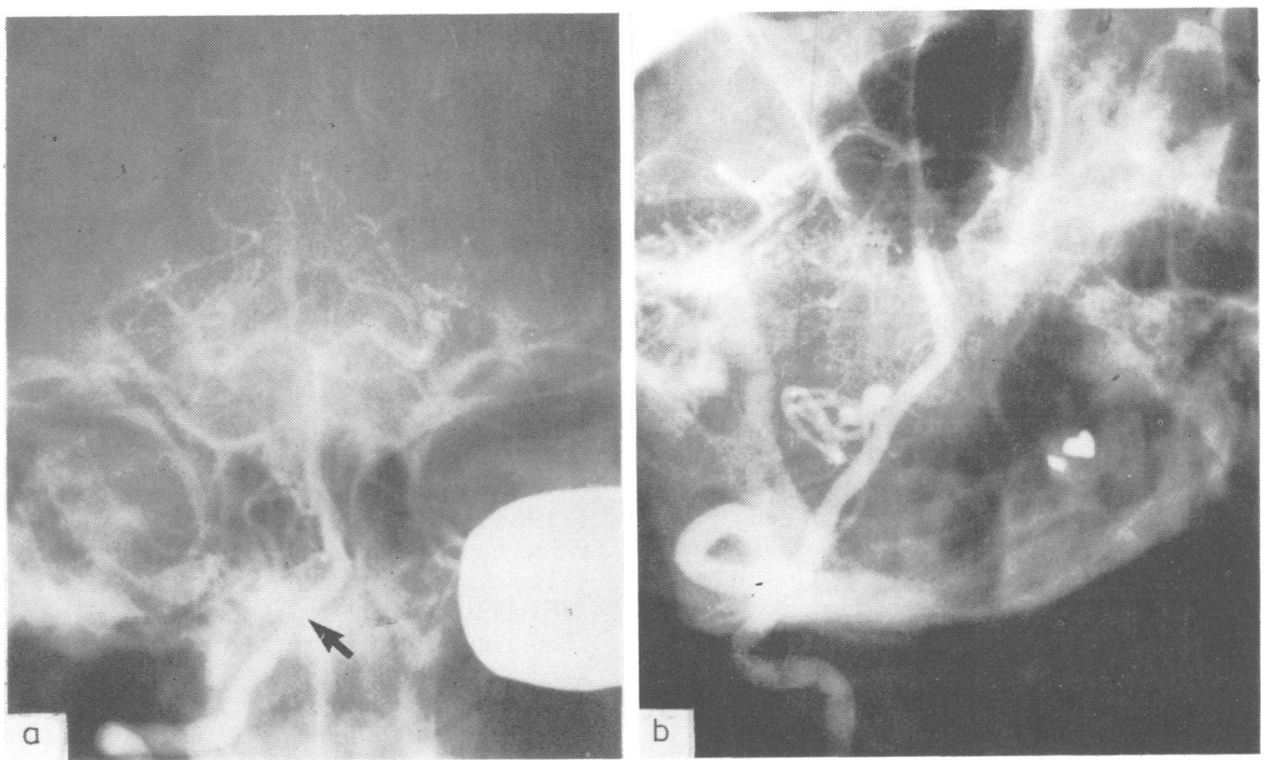

Fig 1 (a) Antero-posterior projection of right vertebral artery angiogram showing the aneurysm arising from the origin of the posterior inferior cerebellar artery (arrow). The distal vertebral artery sweeps beyond the midline to the left of the clivus bringing the neck of the aneurysm to the midline. (b) Oblique projection of the vertebral angiogram reveals the irregularly-shaped fundus and the site of origin of the aneurysm.

of the midline of the medulla, it was decided to attempt a transoral-transclival approach rather than a sub-occipital one. Because pre-operative bacteriological swabs of nose and throat yielded a growth of Staphylococcus aureus, prophylactic flucloxacillin was administered prior to surgery. Antibiotic cream was applied to the nostrils and mouth washes of dilute hydrogen peroxide commenced 48 hours before surgery. After induction the endotracheal tube was strapped to the angle of the mouth and a special retractor, originally developed for transoral intervertebral fusion procedures was inserted and the tongue depressed by a special attachment. A throat pack was inserted and the soft palate incised in the midline and separated for a short distance on each side from the hard palate. Each flap thus created was retracted into the posterior nasopharynx by a stay suture passed through the end of a small Foley catheter previously inserted through each nostril. In this way the flaps were retracted out of the operative field. A posterior pharyngeal mucoperiosteal flap was then $\mathrm{cut}^{8}$ and retracted upwards to expose the clivus and arch of the atlas. Considerable difficulty was experienced in forming this flap because of the very thin pharyngeal musculature. Lateral radiographs were taken at this point to control the line of approach to the clivus. A window was then cut in the bone using a combination of drill and upcut bone punches, taking the anterior part of the rim of the foramen magnum. Having exposed the dura at this point, which was thickened through fusion of the dura with the apical ligaments of the dens, an incision was made creating a $\mathrm{U}$-shaped flap based inferiorly to expose the anterior surface of the medulla. Venous bleeding from dural veins was not a major problem. A solid haematoma obscured the vessels but once this was sucked away a clear view of the right vertebral artery and the origin of the posterior inferior cerebellar artery with the neck and fundus of the aneurysm and the proximal basilar artery was obtained. The fundus now appeared tri-lobular and partly embedded in the anterior surface of the medulla. The medial part of the fundus had to be dissected from an adherent vertebral artery and the neck reduced by bi-polar coagulation before it could be satisfactorily occluded with a straight Sugita clip. The dural flap was replaced, sutured using $9 / 0$ nylon and the defect packed with oxycel. The mucoperiosteal flap was replaced in position with difficulty and sutured carefully using fine Vicryl sutures. The soft palate was replaced and sutured with Vicryl and a postnasal pack inserted to promote adhesion of the mucoperiosteal flap to the underlying surfaces. The endotracheal tube was not removed for the first 24 hours of the post-operative period as there was considerable oedema and swelling of the tongue. Previous experience with this exposure for fusion of the atlanto-axial joint suggested that a tracheostomy would not be necessary. This indeed proved the case. Her immediate post-operative recovery was rapid and she was able to walk five days after surgery. Unfortunately her subsequent post-operative course was complicated by the breakdown of the palatal and posterior pharyngeal suture line and the development of a cerebrospinal fistula.

Fifteen days after surgery she became drowsy with signs of meningitis, confirmed by lumbar puncture. Bacteriological analysis yielded a culture of Klebsiella species resistant 
to a wide range of antibiotics including chloramphenicol, gentamycin and sulphonamides. However it was sensitive to cefotaxime and as this antibiotic penetrates well into the brain and cerebrospinal fluid it was given in high doses by parenteral injection. Fortunately the cerebrospinal fluid fistula closed as the posterior pharyngeal wall healed and the meningitis responded to the antibiotic treatment. During this period repeat CT scans showed a generalised hydrocephalus which was treated by the insertion of a Rickham reservoir and external ventricular drainage. Nasogastric and parenteral nutritional support were given during this period. Oral diet was resumed 2 months after the subarachnoid haemorrhage. A vertebral angiogram carried out 3 weeks later showed that the flow was reduced but the clip appeared in good position and the aneurysm did not fill. Her recovery continued and she was discharged ambulant and orientated without neurological deficit.

Reviewed 5 months later she was without neurological deficit and a simple palatal prosthesis allowed her to drink normally and greatly diminished the nasal quality in her speech.

\section{Discussion}

Although Drake ${ }^{7}$ on the basis of one death out of two patients has abandoned the transoral-transclival route and advocates the sub-occipital approach, the mortality and morbidity for large aneurysms is still high. ${ }^{9}$ This alone justifies consideration and further experience with the transclival route which gives excellent visualisation of the vessels and aneurysm neck. A number of authors ${ }^{8-15}$ have reported aneurysm cases treated by this route, though the technique has not gained wide acceptance. The major problems of cerebrospinal fluid fistula and meningitis remain, and in our experience the recent technique of mucoperiosteal flap advocated by Hayakawa $e t a l^{8}$ and Litvak $e a^{16}$ did not prove entirely satisfactory. It is our intention on a future occasion to use the midline incision which we have used for the transoral atlanteal fusion without complications. Splitting of the soft palate is probably only necessary in patients with small mouths and otherwise should be avoided. Access was aided by a specially-designed retractor but a simple Boyle's mouth gag could be used. Instruments at least $20 \mathrm{~cm}$ in length are essential.

We are grateful to Dr Richard West for angiography and Dr John Turner for general anaesthesia.

\section{References}

' McCormick WF, Notzinger JD. Sacular intracranial aneurysm. An autopsy study. J Neurosurg 1965; 22:155-9.

${ }^{2}$ Locksley HB. Report on the co-operative study of intracranial aneurysms and subarachnoid haemorrhage. Section V, Part 1. Natural history of subarachnoid haemorrhage, intracranial aneurysms and arteriovenous malformations. J Neurosurg 1966; 25:219-39.

${ }^{3}$ Sahs AL, Perret GE, Locksley HB, Nishioka H. Intracranial aneurysms and subarachnoid haemorrhage. A co-operative study. Philadelphia, JI Lippingott, 1969.

${ }^{4}$ Hook O, Norlen G, Guzman J. Sacular aneurysms of the vertebro-basilar arterial system. Acta Neurol Scand 1963;39:271-304.

5 Gillingham FJ, Donaldson AA, Brown AS, MacCabe JJ. The surgical treatment of ruptured basilar aneurysms. Excerpta Medica International Congress Series No 60. Amsterdam: Excerpta Medical Foundation, 1963; Abstract 61.

6 Troupp H. The natural history of aneurysms of the basilar bifurcation. Acta Neurol Scand 1971;47:350-6.

${ }^{7}$ Drake CG. Treatment of aneurysms of the posterior circulation. Clin Neurosurg 1979;26:96-144.

${ }^{8}$ Hayakawa T, Kamikawa K, Ohnishi T, Yoshimine T. Prevention of post-operative complications after transoral/transclival approach to basilar aneurysms. Technical Note. J Neurosurg 1981;54:699-703.

${ }^{9}$ Drake CG. Management of cerebral aneurysms. Stroke $1981 ; 12: 273-83$.

${ }^{10}$ Sano K, Jinbo M, Saito T. Vertebro-basilar aneurysms with special reference to the transpharyngeal approach to the basilar artery aneurysm. No To Shinkei 1966;18:1197-1203.

1 Yasargil MG. Microsurgery Applied to Neurosurgery. Stuttgart: George Thieme Verlag, 1969.

${ }^{12}$ Hashi K, Hakuba A, Ikuno H, et al. A midline vertebral artery aneurysm operated via transclival approach. No Shinkei Geka 1976;42:183-9.

${ }^{13}$ Saito I. Clipping of vertebro-basilar aneurysm by the transclival approach. Neurol Med Chir (Tokyo) Supplement 18, 1978; 175.

14 Yamaura A, Makino H, Isobe K, et al. Repair of cerebrospinal fistula following transoral/transclival approach to a basilar aneurysm. Technical Note. $J$ Neurosurg 1979;50:834-6.

15 Pia HW. Transoral transclival approach. In: Pia HW, Langmaid C, Zierski J, Eds. Cerebral Aneurysms: Advances in Diagnosis and Management. Heidelberg, Springer Verlag 1979:329-31.

${ }^{16}$ Litvak J, Summers TC, Barron JL, Fisher LS. A successful approach to vertebro-basilar aneurysms. Technical Note. J Neurosurg 1981;55:491-4. 